\title{
Predictive police patrolling to target hotspots and cover response demand
}

\author{
Johanna Leigh $^{1}$ - Sarah Dunnett ${ }^{1}$ - Lisa Jackson ${ }^{1}$
}

Published online: 25 May 2017

(C) The Author(s) 2017. This article is an open access publication

\begin{abstract}
Police forces are constantly competing to provide adequate service whilst faced with major funding cuts. The funding cuts result in limited resources hence methods of improving resource efficiency are vital to public safety. One area where improving the efficiency could drastically improve service is the planning of patrol routes for incident response officers. Current methods of patrolling lack direction and do not consider response demand. Police patrols have the potential to deter crime when directed to the right areas. Patrols also have the ability to position officers with access to high demand areas by pre-empting where response demand will arise. The algorithm developed in this work directs patrol routes in real-time by targeting high crime areas whilst maximising demand coverage. Methods used include kernel density estimation for hotspot identification and maximum coverage location problems for positioning. These methods result in more effective daily patrolling which reduces response times and accurately targets problem areas. Though applied in this instance to daily patrol operations, the methodology could help to reduce the need for disaster relief operations whilst also positioning proactively to allow quick response when disaster relief operations are required.
\end{abstract}

Keywords Predictive policing - Data analysis - Kernel density estimation - Resource allocation

\section{Introduction}

Resilience to known and unknown future human induced events, such as crime, in a constantly evolving and changing environment is paramount within the emergency services. Having the ability to preempt these future crimes and to quickly react to any incidents that occur is fundamental to the potential impact, and ultimate consequences, on public safety. Being able

Johanna Leigh

J.M.Leigh@lboro.ac.uk

1 Aeronautical and Automotive Engineering Department, Loughborough University, Stewart Miller Building, Loughborough, Leicestershire LE11 3TU, UK 
to position resources, such as police officers, in the right place at the right time, given the information and knowledge available is key to deterring incidents and also enables the timely arrival at the incidents that do occur.

Having such capabilities is becoming increasingly challenging due to funding cuts being imposed on the force (Newburn 2015). The efficiency of this service can be improved by positioning officers in a configuration which is most suited to respond to incidents and by reducing the number of incidents requiring response. Both of these can be achieved through using predictive policing to direct patrols.

Police officers allocated to response, response units, are directed to incidents by dispatchers. When the response units are not attending incidents their duty is to patrol to deter crime. It has previously been proven that the presence of an officer in high crime areas, known as hotspots, reduces crime levels (Smallwood 2015; Sherman and Weisburd 1995) Alongside deterring crime officers on patrols should also be positioned in a configuration which allows them to reach possible demand within response time targets. Currently within police forces there is limited direction given to where response units should patrol. The current implemented method gives an area of concern with regards to level of incidents and an active time period of approximately $3 \mathrm{~h}$ within which response units are asked to patrol the area. This method does not consider real-time variability or positioning proactively for response, pre-disaster positioning. Due to the limited, and decreasing, number of resources it is important that the time available for officers to patrol is used more efficiently. As the efficiency of patrols can be measured on how well they deter crime and how effectively they are positioned to cope with response demand, post disaster response, a method of directing patrols to target hotspots and position proactively for response using real-time information is proposed in this research.

The research documented in this paper provides a method of directing patrol routes to target crime hotspots and ensure maximum coverage of demand. Initially in the patrol direction process, hotspots are identified using historical police data and performing kernel density estimation mapping. The accuracy of hotspot predictions is increased through using weighting systems. The hotspot areas which provide the highest potential to deter crime are identified. These are the areas where patrolling will be most effective however due to limited resources not every hotspot can be patrolled at once. The best combination of hotspots to patrol, with the available resources at that time, is chosen based on which combination provides the maximum coverage of predicted demand, hence tackling elements of pre and post incident response. This is determined using a version of the maximum coverage location problem (MCLP) designed to solve the police positioning problem. Here the demand coverage of each combination of hotspots is analysed and the best solution is found within the available time. The demand coverage is measured by the availability of response units to reach the predicted demand within the target response times.

Hotspots and demand are continuously changing and hence the problem is dynamic, for that reason real-time solutions to the positioning problem are sought. Due to the time constraints applied from solving in real time a heuristic approach, tabu search, is used. The research has taken place in collaboration with Leicestershire Police in the UK, hence it has benefited from the use of real data and algorithms have been developed to fit with current police procedures.

Along with the policing this method of directing patrols can also be used in other security agencies to direct operations. These agencies face major concerns such as terrorism (Security Service MI5 2017) and human trafficking (National Crime Agency 2016). Using historical data these types of crime can be targeted through operations directed using the patrol direction method detailed here. Security services can proactively direct operations for these types of 
crimes by targeting hotspots for the concerned crime. The action of positioning to deter crime is pre disaster relief operations (DRO) as this takes preventative action against crimes occurring. The action of proactively positioning for incident response is to assist with post DRO by providing quick response.

\section{Related studies}

Previous research in this area can be broken down into work into pre planning patrols to be directed to either deter crime (Chawathe 2007; Li and Keskin 2014) or work into positioning patrolling officers proactively for response (Curtin et al. 2010). This work is discussed below. We believe that there is a gap in the research for a patrolling method which addresses both the deterring crime and incident response and does so considering real-time variability in resources. Before detailing the positioning method proposed related studies which have led to this method are outlined in this section.

As mentioned previously, crime hotspots are an important factor when determining patrol routes, as hotspot targeting is a successful method of reducing crime. The locations in which crimes occur is not stochastic, but is influenced by the geographical layout of an area, as certain area attributes are attractive to criminals such as repeating house design (Chainey and Ratcliffe 2005). This means that when the area is disrupted by an officer patrolling crime can be stopped rather than displaced, hence the overall crime rate falls. Identifying hotspots is common practice within police forces. This started simply with pins on a board to represent crimes and picking out dense clusters of pins as high crime areas. Over the year methods have been enhanced and now include such techniques as spatial ellipses, thematic mapping and kernel density mapping.

Spatial ellipses find areas of high crime density and plot standard deviation ellipses over these areas, hence each ellipse is considered as a hotspot. An example of how this method was utilised to study the effects of school holidays on crime distribution in New York can be seen in Langworthy and Jefferis (2000). The main advantage of this method is that it is not affected by boundaries. The disadvantage is that crime does not occur in areas defined by ellipses.

Thematic mapping requires boundaries such as census areas, police beats or a grid. Each section is then shaded in a colour based on the density of crime in that section. Hotspots are identified by setting a threshold crime level value and areas above this crime level are considered as hotspots. The threshold value is set by the analyst based on the case study. In a study by Chainey et al. (2008) the threshold value was set at $3 \%$ of the total area considered. The use of census areas or police beats as boundaries has the disadvantage of unequal section size, creating a biased representation of crime density. An example application of this method is given by Chainey (2001) for the analysis and visual depiction of crime patterns and auditing across partnership administrative regions. The disadvantage caused by using unequal boundaries is removed by the use of a uniform grid. The grid cell size is also determined depending on the subject by testing. The study by Chainey and Ratcliffe (2005) uses a grid cell size of $200 \mathrm{~m}$ and the study discussed previously by Chainey et al. (2008) uses a grid cell size of $250 \mathrm{~m}$. Thematic mapping with grid cells has been used by Chainey (2001) to analyse the distribution of emergency calls and violent offences. The use of grids does have limitations as information is lost across cell boundaries.

The final method of identifying hotspots discussed here is kernel density mapping. This method is widely used by police forces as shown by Chainey et al. (2008) and LeBeau (2001). 
It is beneficial as it has better spatial analysis and visual properties than the previously discussed methods. The method has also been proven to have the best predictive properties (Chainey et al. 2008). This method represents crime as a continuous surface (Hart and Zandbergen 2014). Grid cells are used in the analysis but the area surrounding the grid cell is also considered within a certain bandwidth of the cell centre.

Research on police patrolling has been performed by Reis et al. (2006) and Chawathe (2007). Reis et al. (2006) developed a program, GAPatrol, to allow patrol routes to be planned in advance. The program finds hotspots iteratively through the construction of visual maps. Multiagent-based simulation is then used to design patrol routes which attend these hotspots more regularly than previous patrols. This work enable patrol routes to be planned that are beneficial to deterring crimes but it does not consider positioning officers so that they can respond to incidents efficiently. In Chawathe (2007) patrols are planned based on two factors. Firstly travelling on roads with higher crime ratings, hence deterring crime, and secondly keeping the cost of travelling low. This study works for a single officer but does not consider multiple officers on duty. It also has the same limitation as the work by Reiss et al., in that it doesn't consider demand coverage.

Other emergency services such as the ambulance service and fire service have a similar problem when positioning their resources for demand. There has been extensive research in this area, some of which is relevant to the police positioning problem. However there is a major difference between these services and the police as ambulances and fire engines are not required to be visible. Their positioning relies solely on where their demand originates from, while police officers must be visible to deter crime and improve the public's feeling of safety. Most ambulance position problems are solved as maximum coverage location problems (MCLP). Research carried out by Daskin and Stern (1981) developed an initial method of ambulance positioning using the basic MCLP principles to position ambulances at fire stations and service stations. This was solved as a stationary problem with no repositioning when ambulances were moved. Future studies were developed based on this method, including a double standard model by Gendreau et al. (1997). The method considers two response time restrictions; it aims to cover a certain percentage of emergency calls within these response times and the rest do not meet response times. This method also considers that different levels of coverage are required, which is important to police positioning, as in areas of high demand one officer is not enough. Hence aspects of the approach adopted in this work for ambulance positioning will be used to solve the police positioning problem. The objective function used by Gendreau for the problem is shown in Eq. 1. The equation represents demand points at a node $i$ by $\lambda_{i}$ and uses $x_{i}^{2}$ to indicate whether $i$ is covered. $x_{i}^{2}$ is a binary number which equals 1 if the demand vertex $i$ in set $V$ is covered a minimum of two times within radius $r_{l}$ and 0 if it is not covered. Constraints are used to set time restrictions.

$$
\text { Maximise } \sum_{i \in V}\left(\begin{array}{ll}
\lambda_{i} & x_{i}^{2}
\end{array}\right)
$$

The research identified has contributed to the development of the patrolling model detailed in this research. The method combines the two processes of identifying hotspots and allocating resources to maximise response demand coverage. The following sections will outline the problem faced by the police and how this problem has been addressed. 


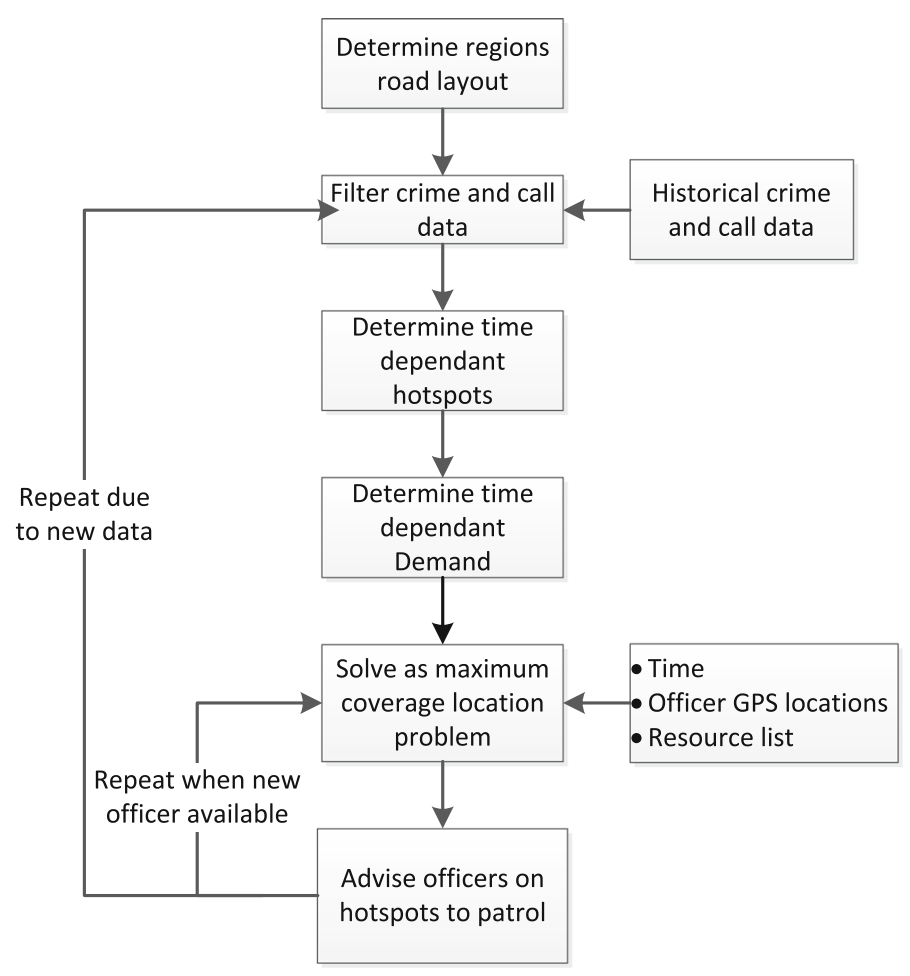

Fig. 1 Flow chart of the main steps in the algorithm developed

\section{Police positioning problem formulation}

Presently patrols are decided based on officer's decisions. The algorithm developed here aims to direct patrols to reduce crimes and be in a better position to react to emergency calls. The algorithm initially identifies hotspots. It does this by taking historical crime data, filtering it in order to discard data not relevant to the problem, and performing kernel density estimation. It then uses these hotspots as possible locations to send officers. Which hotspots are chosen is determined by finding the configuration with maximum coverage of possible demand, using historical call data to predict the demand. The method adopted to calculate the optimal configuration of hotspots to patrol is a version of the MCLP. Performing this analysis once does not give a long-term solution to the patrolling problem as it is a dynamic problem. The location of hotspots and demand are time dependant, as is officer availability. Also once a hotspot has been patrolled the effect of deterring crime in the area has a finite lifetime and hence the area may need to be visited again. Figure 1 shows the main steps in the algorithm developed.

In order to demonstrate the steps outlined a case study has been taken. This is based on Leicestershire though the techniques outlined can be used for any area. The road layout is based on data from OpenStreetMap and is plotted as a directed graph as shown in Fig. 2. 


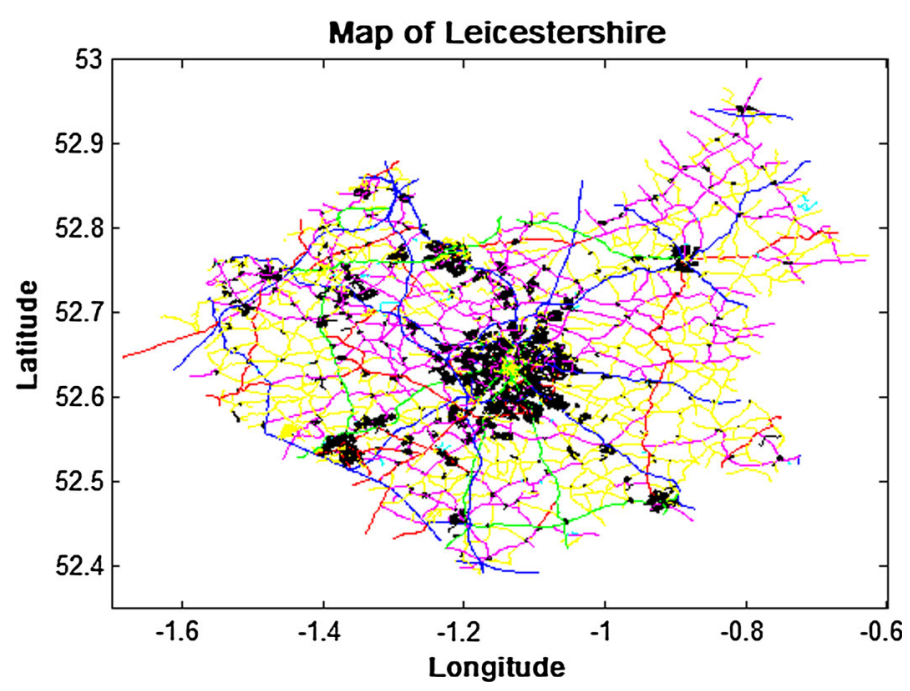

Fig. 2 Road map of Leicestershire

\section{Data analysis}

Police record information on calls made to the force and on crimes which have occurred. This information can be used to inform future policing activities, this is part of evidence based policing. Call data can be used to determine where response demand originates from and this in turn can be used to determine where best to place resources to cope with possible demand. Crime data can be used to determine where crime levels are high and this information can be used to target problem areas with patrols.

Initial filtering of the police data removes repeat crimes and those not recorded properly such as those mapping to a default location instead of where the incident actually occurred. Further filtering narrows down the data set to that which is appropriate to analyse. In the case of call data, each call recorded by the police is given a grading depending on the severity of the incident. The two highest priorities are grade 1 and grade 2 . These require a timely response; hence they contribute to the demand placed on response officers. Therefore the only call data considered when predicting demand will be from grade 2 and grade 1 incidents. Similarly the crime data is filtered to pick out the relevant information to determine the crime hotspots. Only certain crimes can be deterred by the presence of an officer on the street, these include anti-social behaviour, burglary, theft and theft from cars. Hence those crimes not deterred by an officer's presence were removed from the data.

An analysis of the data shows that crime levels vary with the day of the week and time of the day. An example of this is shown in Fig. 3 where the levels of anti-social behaviour (ASB) throughout the day, for the different days of the week, are shown. It can be seen from the graph that the highest levels of ASB are experienced between 1800 and $2000 \mathrm{~h}$. Hence, this is the main time that ASB problem areas should be targeted. It can also be seen that the levels of the crime were particularly high on Wednesdays, Fridays and Sundays. Hence demand and hotspots are time dependent and will be varied within the algorithm developed here, based on the day and time. The hotspots for each time period are found using kernel density estimation (KDE). 


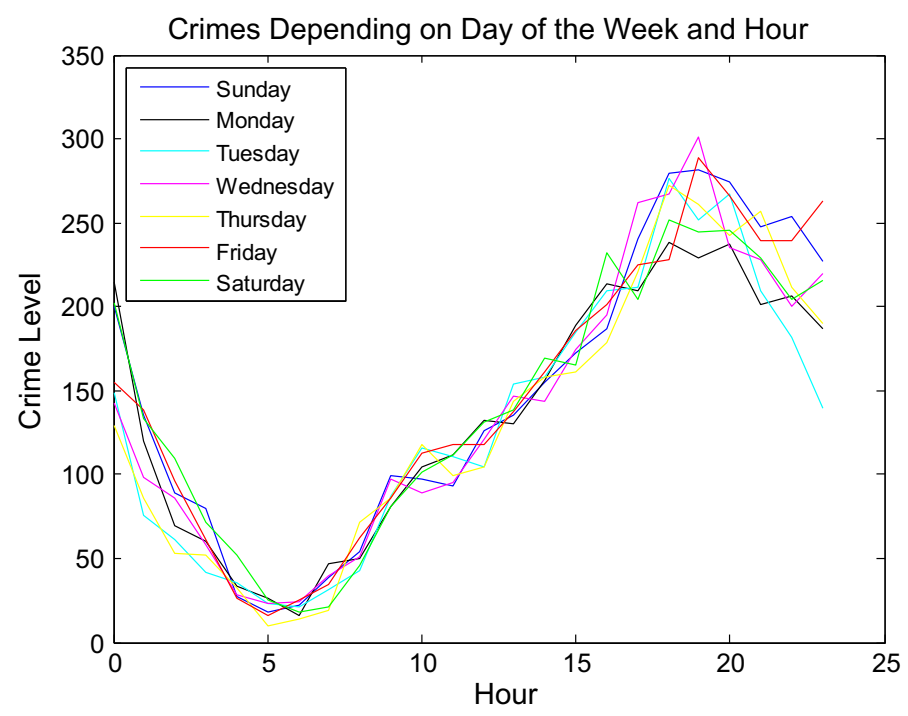

Fig. 3 Variation in ASB depending on time and day

\section{Kernel density estimation}

Out of the many methods of hotspot identification, KDE has been adopted here as it has been proven to be the method with the best predictive abilities (Chainey et al. 2008). This method of analysis reduces the effect of boundaries on the results by taking into account crimes in the surrounding area.

$\mathrm{KDE}$ is performed using a grid overlaid on top of the street map and crime data. In this study the grid consists of $0.001^{\circ}$ by $0.001^{\circ}$ cells. The effect of the grid is reduced, for each grid cell, by considering the crimes within a circle whose centre is at the cell centre and whose diameter (bandwidth) is larger than the grid cell size. All the crimes within this bandwidth are assumed to contribute to the intensity of crimes within the cell. The bandwidth radius used for this study is $0.001^{\circ}$. The grid cell size and bandwidth have been determined through considering the area of interest and testing the effects of using different sizes. The decision is made based on a compromise between accurate identification of hotspot and computational time. The KDE calculation is then performed on each of the grid cells using Eq. 2 taken from Gatrell et al. (1996). This equation finds the intensity of crimes $\left(\hat{\lambda}_{\tau}(s)\right)$ within the bandwidth radius $(\tau)$ as a function of the distance from the cell centre $(S) . d_{i}$ is the distance between the grid centre $(S)$ and the point being investigated $S_{i}$. Figure 4 shows the parameters more clearly. The further the crime from the centre of the grid cell the less it adds to the intensity.

$$
\hat{\lambda}_{\tau}(s)=\sum_{d_{i} \leq \tau} \frac{3}{\pi \tau^{2}}\left(1-\frac{d_{i}^{2}}{\tau^{2}}\right)^{2}
$$

Once the crime intensity in the region is known the hotspots are identified as the areas with the highest $\mathrm{n} \%$ intensity, where $\mathrm{n}$ is dependent on the situation. These hotspots can be used as possible patrol locations. This will target problem areas and help to reduce crime. However, not all of the hotspots can be constantly visited, certain hotspots will be chosen based on what configuration provides the best demand coverage. 


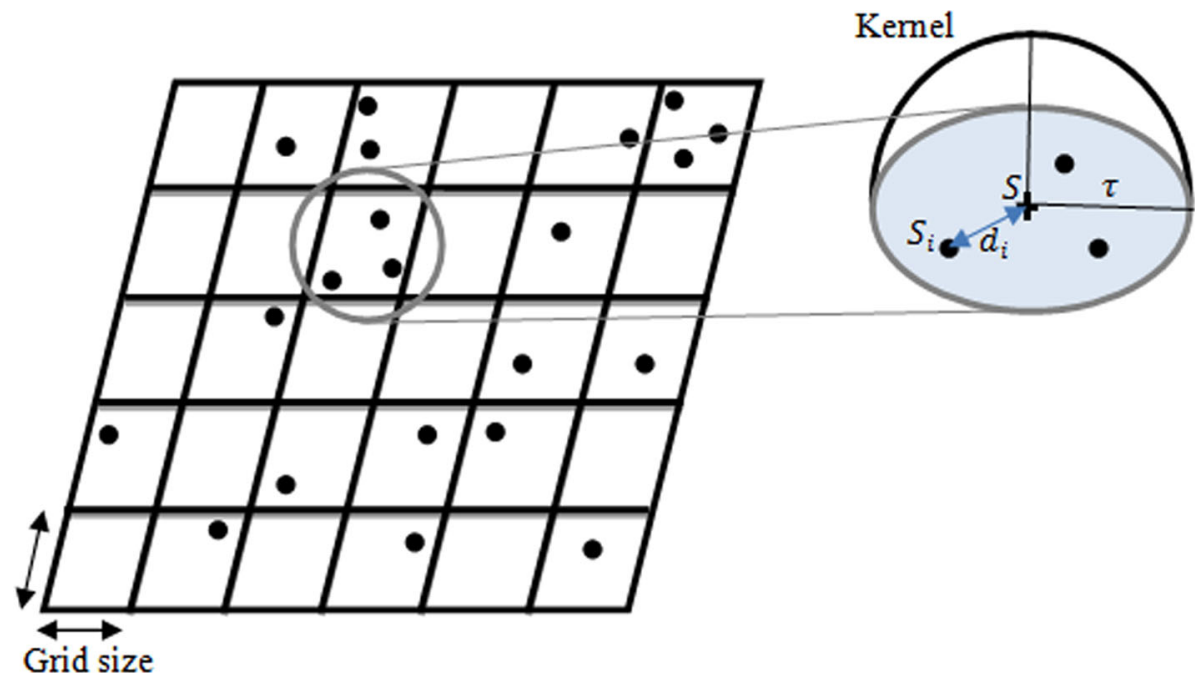

Fig. 4 Kernel density estimation

\section{Maximum demand coverage}

Having determined the hotspots the next stage is to determine the best configuration of these hotspots to allocate resources to, in order to meet possible demand within the emergency response time targets. Demand is calculated from the historical data through overlaying a grid onto the street map and determining the demand level within the grid cells from the call data. This demand is also time dependant, and will change depending on the day of the week and the time of day. Demand is considered covered if an officer can reach the area, containing the demand, within the recommended response time for emergency, grade 1, incidents. In Leicestershire this is $15 \mathrm{~min}$ for populated areas such as cities and town centres and $20 \mathrm{~min}$ for less populated areas such as rural areas. In the algorithm detailed the two response time targets are labelled as $t_{c}$ for cities and towns and $t_{r}$ for rural areas.

To determine which layout of hotspots, when covered by the patrol routes, has the optimal demand coverage the problem can be modelled as a maximum coverage location problem (MCLP) such as used in ambulance dispatch. In this case the hotspots are the possible locations where officer's patrol, represented by $W=\left\{w_{1}, w_{2}, \ldots, w_{m}\right\}$ where each of these are a grid cell and $\mathrm{m}$ is the total number of hotspots found during the kernel density analysis. The demand points are the centres of each of the grid cells and are represented by $V=$ $\left\{v_{1}, v_{2}, \ldots, v_{n}\right\}$ where $\mathrm{n}$ is the total number of cells. These points are then used in a variation of the MCLP based on that used for the ambulance positioning problem by Gendreau et al. The variation considers the two response time standards using the following rules for demand point $\mathrm{v}_{\mathrm{i}}$ :

1. If $v_{i}$ is in a city, or town, centre it is only considered covered if a unit can reach the point within the time period $t_{c}$. In this case it is assumed that a response unit can cover the distance $r_{1}$ within the time $t_{c}$.

2. If $v_{i}$ is in a rural area it is considered covered if a unit can reach it within the time period $t_{r}$. In this case it is assumed that a response officer can cover the distance $r_{2}$ within the time $t_{r}$.

3. $r_{1}<r_{2}$. 
In the case study considered here the maximum average speed which an officer can travel to reach an incident is 50 miles per hour. This results in $r_{1}=20 \mathrm{~km}$ and $r_{2}=27 \mathrm{~km}$ being the maximum distance an officer can be from a demand point, in city and rural areas respectively, for it to be considered covered.

The objective function devised for this problem is shown in Eq. 3. It looks at each demand point, $v_{i}$, determines which region it's in, city or rural, and whether the demand at that point is covered. $C$ and $R$, denoting city and rural, are binary values where 1 signifies the point is within that classification and 0 signifies that it is outside. In Eq. (3) ${ }_{j} x_{i}^{k}$ is a binary value which equals 1 if the demand point $v_{i}$ is covered a minimum of $\mathrm{k}$ times within the radius $r_{j}$ and $\lambda_{i}$ is the demand at that point.

$$
\text { Maximise } \sum_{i \in V}\left(\begin{array}{lll}
\lambda_{i} & 1 & x_{i}^{k} C+\lambda_{i} \quad{ }_{2} x_{i}^{k} R
\end{array}\right)
$$

The objective function is subject to the constraints shown in Eqs. (4)-(9).

$$
\begin{aligned}
& \sum_{j \in W} y_{j}=p \\
& { }_{1,2} x_{i}^{k+1} \leq 1,2 x_{i}^{k}\left(v_{i} \in V\right) \\
& { }_{1} x_{i}^{k},{ }_{2} x_{i}^{k} \in\{0,1\}\left(v_{i} \in V\right) \\
& C, R \in\{0,1\} \\
& C+R=1 \\
& r_{w} \leq r_{s}
\end{aligned}
$$

In these equations $y_{j}$ represents the number of resources located at hotspot $w_{j}$. The total number of units available is taken to be $p$, constraint (4), and this is determined by the number of officers on shift with an available status at that time, and whether they are single or double crewed. Constraint (5) states that node $v_{i}$ can only be covered $k+1$ times if it is covered at least $k$ times. Constraint (6) and (7) ensures ${ }_{1} x_{i}^{k},{ }_{2} x_{i}^{k}, C$ and $R$ are binary numbers. Constraint (8) states that demand is either in a city or rural area but never both at the same time. Each police officer has a station which they are based from. When allocating patrol activities to officers they cannot be allocated a region too far from their base station. This is represented by constraint (9) where $r_{w}$ is the distance from a units base police station to the possible patrol location for the unit and $r_{s}$ is the maximum distance the unit is allowed to be assigned from their base station, determined by the police force. When solving the MCLP there are other constraints laid out by the police, these are;

- a unit can only be repositioned if their status is available,

- a unit whose status is unavailable because they are attending to non-emergency incidents can still contribute to demand coverage. However they cannot be moved for other purposes.

\section{Searching for solutions}

In determining the hotspots to be patrolled that give maximum demand coverage there are many possible combinations to consider. To find the optimal solution an exhaustive search can be performed and the solution with the highest demand coverage chosen. This search method has high computational costs. For the application being considered here the demand and 
hotspots are continually changing and hence the problem has to be solved many times. This makes exhaustive search infeasible and hence other methods have been investigated. One of these, tabu search, allows the search area to be narrowed resulting in a lowered computational time to obtain a solution than exhaustive search. This method does not guarantee finding the optimum solution but it has been shown to be an efficient and accurate way of solving similar problems (Gendreau et al. 1997).

\subsection{Tabu search}

The Tabu search process initially choses a random feasible combination of hotspots, $\mathrm{S}$, and the objective function, Eq. (3), is then obtained to find the demand coverage of that combination, $f^{*}$. The search for a better solution continues using neighbourhood search. Hence the next combinations to be investigated are the neighbours, $\mathrm{N}(\mathrm{S})$, of the previous solution, where a neighbour is one move away from the previous solution. In this case, one move is classed as changing one hotspot in the combination. Out of the neighbouring solutions, the best, $\mathrm{S}^{*}$, is chosen to continue the rest of the search, even if this is worse than the previous combination. Allowing a worse solution to be chosen stops the search getting stuck at a local optima. The best, $\mathrm{S}^{*}$, is classified as the solution which maximises the objective function, $\operatorname{argmax}[f(\mathrm{~S})]$, i.e. the solution which results in the highest demand coverage. $\mathrm{S}^{*}$ is set to the current solution $\mathrm{S}$ and its neighbours are now considered. The search stops when one of the following stopping criteria are met:

- No improvement solution has been obtained for a set number of iterations

- The maximum number of iterations has been reached

- The optimal solution is found, where all points are covered.

Tabu lists are used to stop the process revisiting the same solutions. This is achieved by adding visited hotspot combinations to a tabu list for a set number of iterations ensuring that they are not revisited in that time. Another tabu list with a longer memory is also formed, which contains hotspots that have been visited by a unit. This is because the effect of a unit patrolling a hotspot lasts for some time after they have left the area, hence revisiting immediately is not effective. Also other hotspots which haven't recently been patrolled should be visited before repeat visits are made. The hotspot is removed from the tabu list when revisiting is required. How the algorithm deals with revisiting hotspots is described below.

\subsection{Hotspot revisiting}

A study by Chen et al. (2015) looked at how ant colony algorithms can be used to plan patrol routes. Their study developed a patrolling strategy using Bayesian methods and ant colony algorithms. The paths patrolled by units were marked by virtual pheromone with an exponential decay, and which hotspot to patrol next was determined by the pheromone levels. This is a good method of stopping repeat hotspot visiting within short spaces of time whilst also tracking when another visit is required. It also enables the effect a unit patrolling leaves on an area once they have moved on, to be tracked. The same approach has been adopted in this paper. When pheromone level is high the hotspot is placed on the tabu list and hence is not revisited in a short space of time. Once the level drops below the threshold value the hotspot is removed from the tabu list. The threshold level is determined by the intensity of the hotspot. 


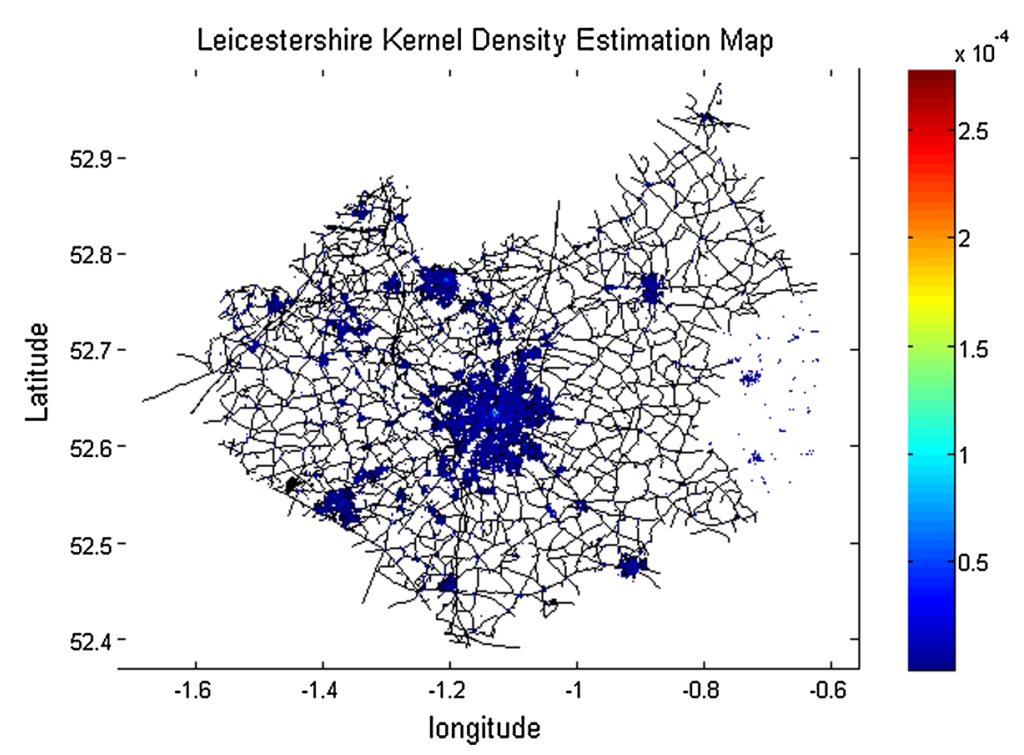

Fig. 5 KDE map for all crimes

\section{Results}

To assess the effects of the algorithm developed on criminal activity agent based simulation has been used. The agents within the simulation are response units; each agent has tasks to perform during their shift. The number of agents within the simulation is based on shift patterns by Leicestershire police. These agents have different states depending on what tasks they are performing, they are classed into two states, available and unavailable. Available means they can be allocated a region to patrol. Unavailable means they are performing other tasks such as attending an incident or on a break. In this work the amount of time that units are available is based on the proportion of a shift that the force perceives units are available to patrol. For this example this is taken to be $5 \%$ of the shift. The time period which the simulation is run over is based on a one month historical period in time.

The first outcomes of the algorithm are the kernel density estimation graphs formed from the historical crime and call data. These are what are used when the system is running in real time. The KDE map produced for overall crime is shown in Fig. 5 for all of Leicestershire. Figure 6a, b show the maps for Leicester city centre and Loughborough town centre, where Loughborough is the largest town in the county outside Leicester city. The colours which the areas are shaded in these figures represent the intensity of crime within that area and the scale is shown on the right of the diagrams. Areas with no shading had no significant crimes within that period. The map of the entire county shows that the crime density increases around towns and cities as expected. The map of Leicester city centre and Loughborough town shows the difference in crime density on a more granular level. Figures 7, 8 and 9 are KDE maps for the crime types; burglary, criminal damage and assault. These show the differences in crime trends for different types of crime. The hotspots appear in different areas in each graphs and therefore analysing the crimes separately will allow certain types of crimes to be targeted. Sexual assault trends, which have not been shown within these results for sensitivity reasons, demonstrate a more spread out dispersion of crime compared to burglary, criminal damage 


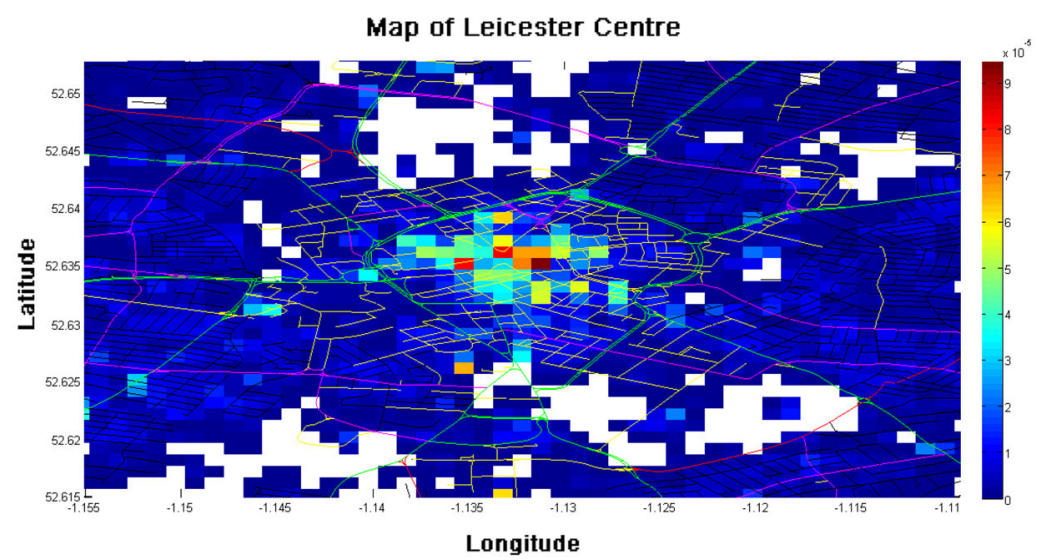

(a)

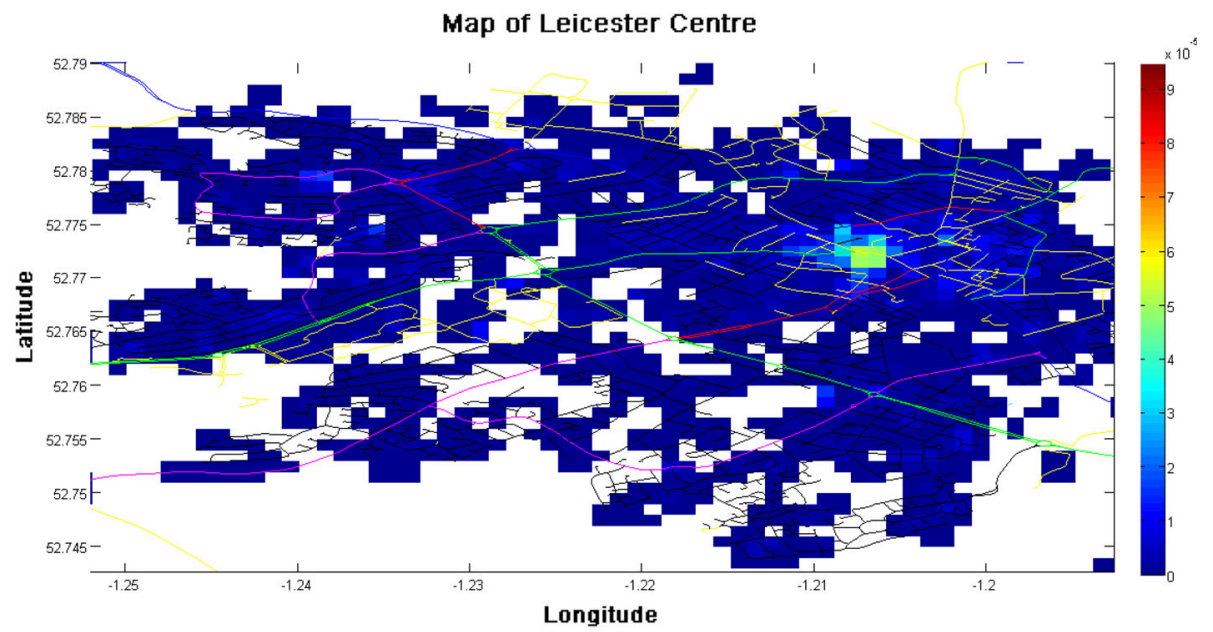

(b)

Fig. 6 KDE map for a Leicester city centre, b Loughborough town

and assault. Of the intensities calculated the highest $3 \%$ are taken to be hotspot regions and used within the MCLP. This means $3 \%$ of the total region being set to hot.

As well as the use of multiple hotspots considering different types of crime it is necessary to use different hotspots at different times of day. Figure 3 has previously shown the variation of crime throughout a day. Figure 10 shows that the location of crimes also changes throughout the day. In this figure the KDE maps for criminal damage are shown for 3 different time periods over all days. The movement in crime hotspots throughout the day can be explained by the attractiveness of an area at different times of day due to the lighting, number of people and amenities open.

As this is a historical period in time the crime and call data for that period is available and hence the effect of placing patrol units in hotspots can be assessed. This is achieved by the use of Eq. (10), which was used in the study by Chainey et al. (2008) to determine the predictive performance of different hotspot identification methods. It is used in this study 


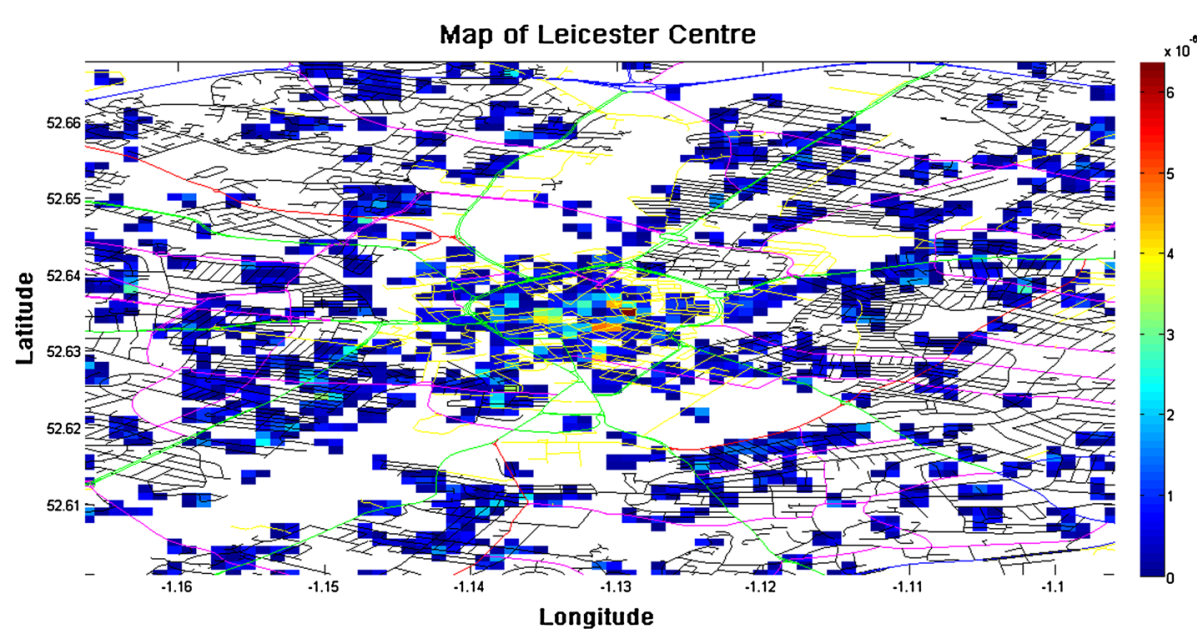

Fig. 7 Burglary KDE map for Leicester city centre

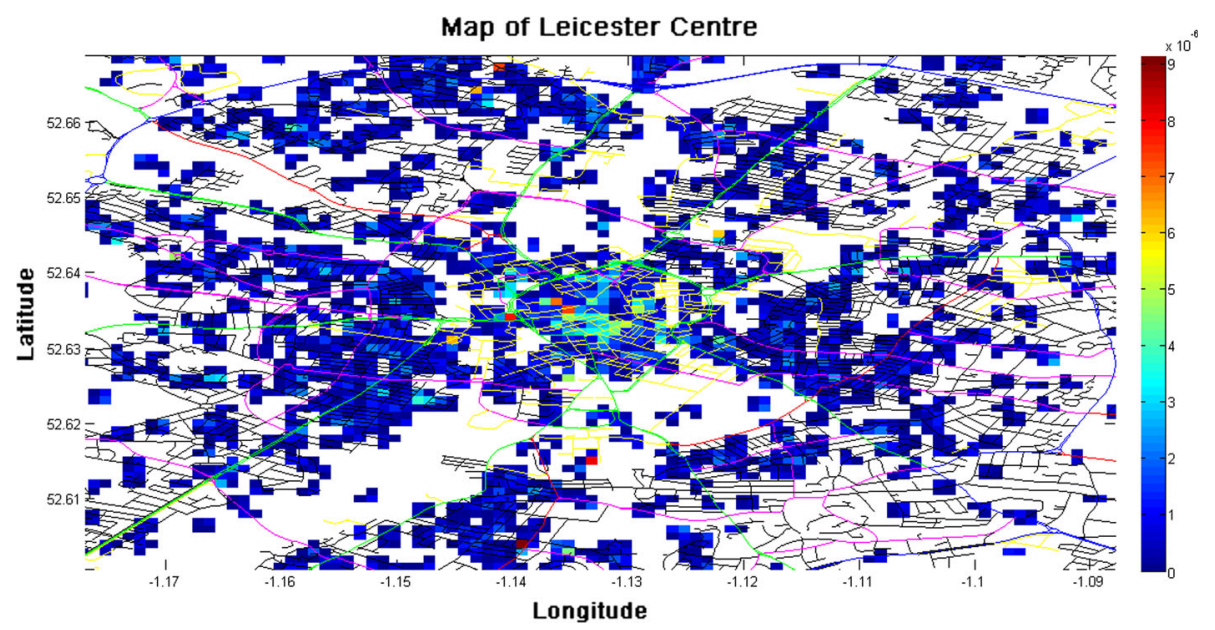

Fig. 8 Criminal damage KDE map for Leicester city centre

to determine the level of crime the directed patrol routes have the ability to deter. In the equation $\mathrm{n}$ represents the number of crimes which occur within the hotspots targeted by patrolling, whilst $\mathrm{N}$ is the total number of crimes which occur, a is the total area of all the hotspots targeted, whilst $\mathrm{A}$ is the total area of the region studied. The results obtained from the simulation shows a potential to decrease street crime by $22 \%$ when the simulation is run over a month.

$$
\frac{\frac{n}{N} \times 100}{\frac{a}{A} \times 100}=\frac{\text { Hit Rate }}{\text { Area Percentage }}=\text { Targetting Accuracy Index }
$$

An example of a typical spatial demand layout for a section of Leicestershire is shown in Fig. 11. The demand profile is shown in Eq. 11 as a matrix. Each element in the matrix represents the number of incidents which required a timely response within the related historical period in the related grid cell. This method is used to analysis the demand profile for the 


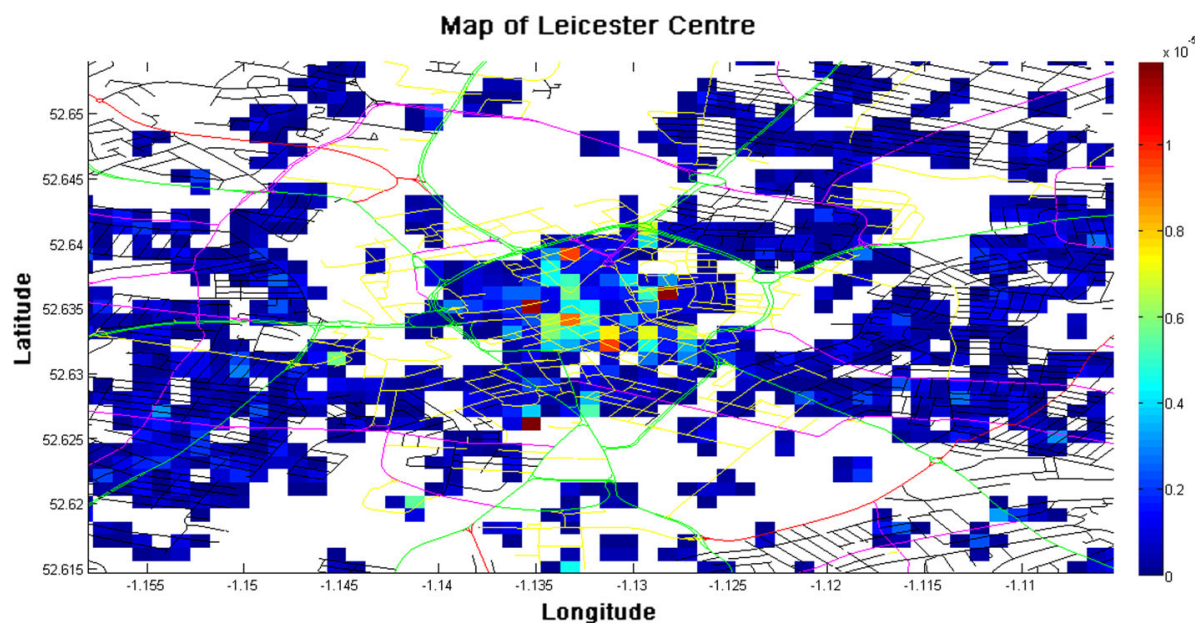

Fig. 9 Assault KDE map for Leicester city centre
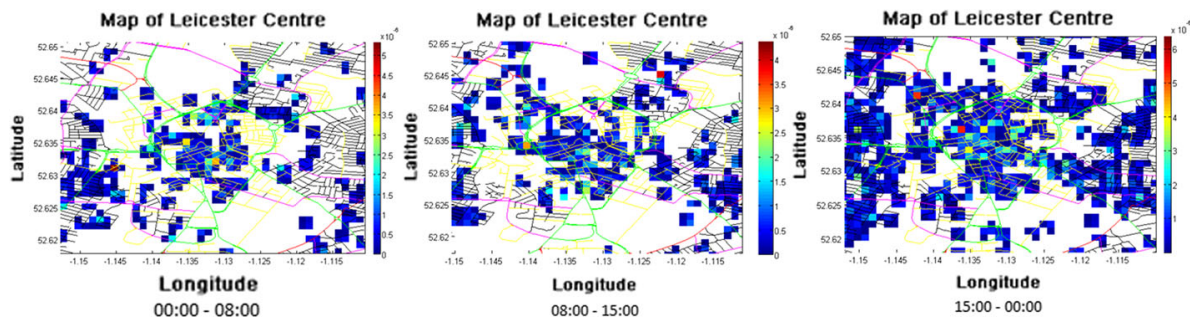

Fig. 10 Time dependant criminal damage hotspots

entirety of Leicestershire and the results are used in the simulation when determining the response demand coverage each configuration of officers results in.

$$
D=\left[\begin{array}{llllll}
3 & 1 & 2 & 0 & 2 & 0 \\
1 & 4 & 1 & 0 & 0 & 0 \\
0 & 0 & 2 & 1 & 0 & 2 \\
1 & 2 & 1 & 1 & 2 & 0 \\
0 & 1 & 1 & 0 & 0 & 1
\end{array}\right]
$$

Discrete event simulation is used to test the ability of the maximum coverage algorithm to position officers, using the tabu search method as described above. Solving MCLP for one set of positions takes on average $74.38 \mathrm{~s}$ each time the problem was solved. Initially all hotspots are considered as visited and as time passes they each require revisiting. The demand coverage results can be seen in Table 1 . This shows the minimum coverage achieved, the average coverage achieved, the maximum coverage achieved and the average change in coverage from the original positions of officers to the directed positons. The minimum case was due to limited resources. The maximum case was a low crime level time resulting in most officers being available to patrol. The average change shows that using the positioning algorithm can result in a 2.13 change in response demand coverage which is almost a $50 \%$ increase from the original officer positions. 


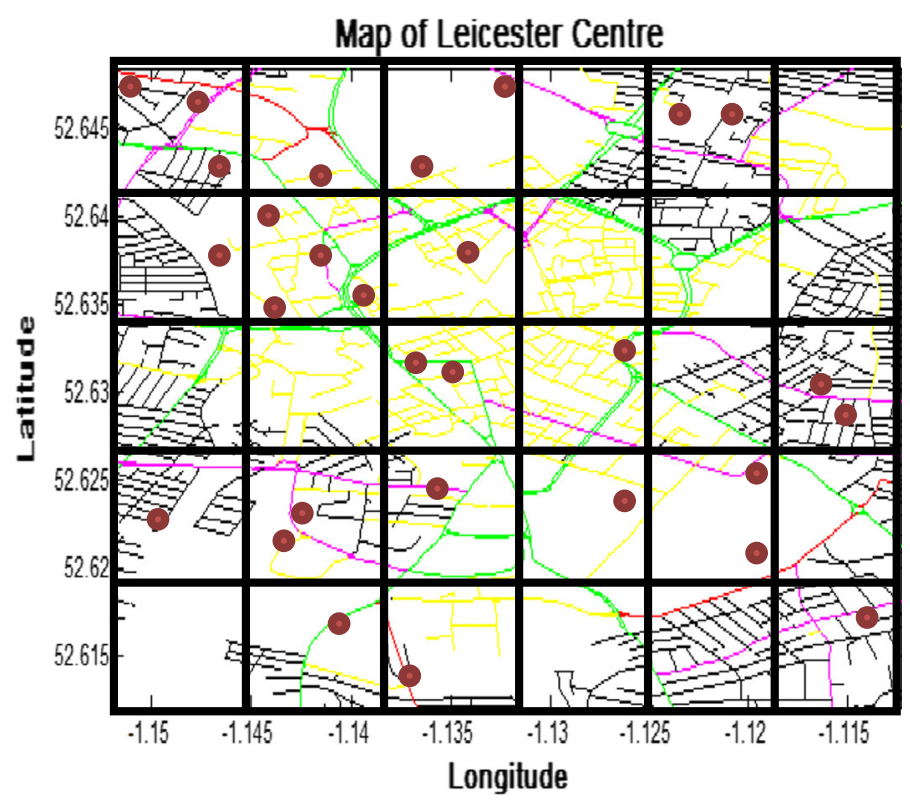

Fig. 11 Response demand analysis

Table 1 Results of demand coverage

\begin{tabular}{ll}
\hline & Demand coverage $\left(\mathrm{km}^{-2}\right)$ \\
\hline Minimum & 0.26 \\
Average achieved & 4.42 \\
Maximum & 6.72 \\
Average change & 2.13
\end{tabular}

The increase in demand coverage will allow response times to be reduced when it is implemented. The decrease in response times allows officers to be more effective, provide a better service for the public which improves the public's perception of the police. The decrease also results in higher availability of officers.

\section{Conclusion}

This research addresses the issue currently faced by police forces of inefficient patrolling. Improving these patrols by directing them to problem areas could have major benefits on policing including reducing crime levels. The real time aspect of the algorithm allows officers to be positioned efficiently to respond to possible emergency incidents through demand modelling. This reduces the response times. The study falls in line with the push towards evidence based policing over biased policing which can occur in some regions due to people's opinions. Other uses of the algorithm also include testing the effect of changing officer numbers on demand coverage.

Future work in this area includes testing with a police response department. Initially testing is required to verify the hotspot analysis by targeting the hotspot areas. Further testing will use the algorithm to allocate response units to hotspots. Also development of the algorithm 
into a user friendly program which is compatible with software currently in use by police forces is required. This will allow the methods of directing patrols to be used within any police force. Once this is developed further criteria can be added to patrols such as visiting people of interest on route to patrols.

Acknowledgements The cooperation of the Leicestershire Police is gratefully acknowledged as without this support the project would not be possible. This work was supported by the Economic and Social Research Council [ES/K002392/1].

Open Access This article is distributed under the terms of the Creative Commons Attribution 4.0 International License (http://creativecommons.org/licenses/by/4.0/), which permits unrestricted use, distribution, and reproduction in any medium, provided you give appropriate credit to the original author(s) and the source, provide a link to the Creative Commons license, and indicate if changes were made.

\section{References}

Chainey, S. (2001). Combating crime through partnership: Examples of crime and disorder mapping solutions in London, UK. In A. Hirschfield \& K. Bowers (Eds.), Mapping and analysing crime data: Lessons from research and practice. London: Taylor Francis.

Chainey, S., \& Ratcliffe, J. (2005). GIS and crime mapping. London: Wiley. (chapter 4).

Chainey, S., Tompson, L., \& Uhlig, S. (2008). The utility of hotspot mapping for predicting spatial patterns of crime. Security Journal, 21(1-2), 4-28.

Chawathe, S. S. (2007). Organizing hot-spot police patrol routes. In Intelligence and security informatics, 2007 IEEE (pp. 79-86). IEEE.

Chen, H., Cheng, T., \& Wise, S. (2015). Designing daily patrol routes for policing based on ant colony algorithm. ISPRS Annals $f$ the Photogrammetry Spatial Informatics Sciences, II-4/W2, 103-109.

Curtin, K. M., Hayslett-McCall, K., \& Qiu, F. (2010). Determining optimal police patrol areas with maximal covering and backup covering location models. Networks and Spatial Economics, 10(1), 125-145.

Daskin, M. S., \& Stern, E. H. (1981). A hierarchical objective set covering model for emergency medical service vehicle deployment. Transportation Science, 15(2), 137-152.

Gatrell, A., Bailey, T., Diggle, P., \& Rowlingson, B. (1996). Spatial point pattern analysis and its applications in geographical epidemiology. Transactions of the Institute of British Geographers, 21(1), 256-274.

Gendreau, M., Laporte, G., \& Semet, F. (1997). Solving an ambulance location model by tabu search. Location Science, 5(2), 75-88.

Hart, T., \& Zandbergen, P. (2014). Kernel density estimation and hotspot mapping. Policing: An International Journal of Police Strategies and Management, 37(2), 305-323.

Langworthy, R., \& Jefferis, E. (2000). The utility of standard deviation ellipses for evaluating hotspots. In V. Goldsmith, P. McGuire, J. Mollenkopf, \& T. Ross (Eds.), Analyzing crime patterns: Frontiers of practice (pp. 87-104). Thousand Oaks, CA: Sage Publications.

LeBeau, J. L. (2001). Mapping out hazardous space for police work. In A. Hirschfield \& K. Bowers (Eds.), Mapping and analysing crime data: Lessons from research and practice (pp. 139-155). London: Taylor and Francis.

Li, S., \& Keskin, B. (2014). Bi-criteria dynamic location-routing problem for patrol coverage. Journal of the Operational Research Society, 65(11), 1711-1725.

National Crime Agency. (2016). Modern slavery and human trafficking. National Crime Agency. http://www. nationalcrimeagency.gov.uk/crime-threats/human-trafficking.

Newburn, T. (2015). What's happening to police numbers? BBC News UK. http://www.bbc.co.uk/news/uk34899060.

OpenStreetMaps, \& Contributors, Maps. (2015). http://www.openstreetmap.org/. Accessed September 01, 2014.

Reis, D., Melo, A., Coelho, A., \& Furtado, V. (2006). Towards optimal police patrol routes with genetic algorithms. Mehrotra, 3975, 485-491.

Security Service MI5. (2017). Terrorism-Threat levels. Security Services MI5. https://www.mi5.gov.uk/ threat-levels.

Sherman, L. W., \& Weisburd, D. (1995). General deterrent effects of police patrol in crime "hot spots": A randomized, controlled trial. Justice Quarterly, 12(4), 625-648.

Smallwood, J. (2015). What works crime reduction: Operation SAVVY. College of Policing. http://whatworks. college.police.uk/Research-Map/Pages/ResearchProject.aspx?projectid=306. 